\title{
Imbalance of angiotensin-converting enzymes affects myocardial apoptosis during cardiac arrest induced by acute pulmonary embolism in a porcine model
}

\author{
HONG-LI XIAO ${ }^{1}$, LIAN-XING ZHAO ${ }^{2}$, JUN YANG $^{2}$, NAN TONG $^{2}$, LE AN $^{2}$, \\ QI-TONG LIU ${ }^{3}$, MIAO-RONG XIE ${ }^{1}$ and CHUN-SHENG LI ${ }^{1}$ \\ ${ }^{1}$ Department of Emergency Medicine, Beijing Friendship Hospital, Capital Medical University, Beijing 100050; \\ Departments of ${ }^{2}$ Emergency Medicine and ${ }^{3}$ Radiology, Beijing Chao-Yang Hospital, \\ Capital Medical University, Beijing 100020, P.R. China
}

Received May 13, 2017; Accepted January 15, 2019

DOI: $10.3892 / \mathrm{ijmm} .2019 .4109$

\begin{abstract}
Acute pulmonary embolism (APE) with cardiac arrest (CA) is associated with a high mortality rate. Even upon return of the spontaneous circulation (ROSC), APE-CA survivors are prone to myocardial cell apoptosis, a key cellular mechanism that induces heart failure. A recent study by our group discovered a post-resuscitation imbalance in the serum angiotensin-converting enzyme (ACE)2/ACE axis of the renin-angiotensin system (RAS), as well as regressive cardiac function in a porcine model of APE-CA. However, it has remained elusive how this imbalance in the ACE2/ACE axis affects myocardial cell apoptosis. In the present study, western blot and immunohistochemical analyses demonstrated that the RAS was only activated in the left myocardium, as evidenced by a decreased ACE2/ACE ratio following APE-CA and ROSC, but not the right myocardium. Ultrastructural analysis confirmed myocardial apoptosis in the left and right myocardium. Furthermore, B-cell lymphoma 2 (Bcl-2)-associated X protein (Bax) and caspase-3 levels were elevated and Bcl-2 levels were decreased in the left myocardium following APE-CA and ROSC. Treatment with the ACE inhibitor captopril for 30 min after initiation of ROSC prevented the increase in Bax and the decrease in Bcl-2 in the left myocardium compared with that in saline-treated pigs. Captopril also inhibited the activation of extracellular signal-regulated kinase
\end{abstract}

Correspondence to: Professor Chun-Sheng Li or Professor Miao-Rong Xie, Department of Emergency Medicine, Beijing Friendship Hospital, Capital Medical University, 95 Yongan Road, Xicheng, Beijing 100050, P.R. China

E-mail: 1cscyyy@163.com

E-mail: xiemiao27@126.com

Key words: acute pulmonary embolism, cardiac arrest, return of spontaneous circulation, angiotensin-converting enzyme, myocardial apoptosis
(ERK)1/2 in the left myocardium. The results of the present study suggest that an imbalance in the ACE2/ACE axis has an important role in myocardial apoptosis following APE-CA, which may be attributed to decreased ERK1/2 activation. In addition, it was indicated that captopril prevents apoptosis in the left myocardium after ROSC.

\section{Introduction}

Acute pulmonary embolism (APE) has been frequently reported in critical care and emergency medicine departments and is associated with a high mortality rate. Based on a patient's hemodynamic status and clinical characteristics, APE may be classified as massive, submassive or non-massive (1). Massive pulmonary embolism is life-threatening and is characterized by cardiogenic shock and cardiac arrest (CA) upon presentation (2). The mortality of patients with APE presenting with CA may be as high as $65 \%$ (3). Survivors of such acute events may be more prone to heart failure (4). Myocardial cell apoptosis is one of the underlying key mechanisms associated with heart failure (5). Numerous studies have suggested that the classic angiotensin (Ang)-converting enzyme (ACE)/Ang II/Ang II type 1 receptor axis of the renin-angiotensin system (RAS) regulates hemodynamics, as well as maintains ventricular remodelling (6) and myocardial cell apoptosis (7). By contrast, a newly discovered ACE2/Ang(1-7)/Mas axis within the RAS has been reported to prevent myocardial cell apoptosis caused by myocardial infarction or ischemic reperfusion (8). A recent study by our group reported a post-resuscitation imbalance in the serum ACE2/ACE axis that was accompanied by regressive cardiac function, including lower left heart stroke index, left cardiac work index, left ventricular stroke work index, right cardiac output, right heart stroke volume, right cardiac index and right heart stroke index, in a porcine model of APE-CA (9). However, the role of this imbalance in cardiac tissues during massive APE-CA has remained to be fully elucidated. Therefore, the present study further investigated the association between the imbalance of the cardiac ACE2/ACE axis and myocardial apoptosis in a porcine APE-CA model. 
The previous study by our group indicated that an ACE inhibitor, captopril, lowered the post-resuscitation mean right ventricular pressure and pulmonary vascular resistance through activating the serum ACE2/Ang(1-7)/Mas axis in APE (9). the ability of captopril to inhibit myocardial cell apoptosis during myocardial ischemia/reperfusion injury was previously reported (10). Studies have also suggested that treatment with ACE2, Ang-(1-7) and ACE inhibitors, or with angiotensin receptor blockers, suppresses activation of the extracellular signal-regulated kinase (ERK)1/2 pathway, which is known to have a role in myocardial cell apoptosis $(11,12)$. Therefore, the present study tested the hypothesis that the ACE inhibitor captopril attenuates myocardial cell apoptosis induced by massive APE.

\section{Materials and methods}

Ethical approval. The present study used 29 landrace pigs (age, 3 months; weight, $28 \pm 2 \mathrm{~kg}, 15$ boars and 14 gilts) provided by the Beijing Experimental Animal Center (Beijing, China; license no. SCXK 11-00-002), as described previously (9). All animals were fed standard chow and had free access to water and housed in their own cages with the size of $80 \times 80 \times 90 \mathrm{~cm}$ in our facility. Room temperature was adjusted to $26^{\circ} \mathrm{C}$, and humidity was $60 \%$. Appropriate care was taken to minimize animal suffering. The Committee on the Ethics of Animal Experiments of Capital Medical University approved the study procedures (permit no. 2010-D-013). Experimental protocols were designed in strict compliance with the Animal Care Guidelines of the Institutional Animal Care and Use Committee of Capital Medical University (Beijing, China) as well as the Utstein-style guidelines (13).

Animal preparation. All animals received an intramuscular injection of midazolam $(0.2 \mathrm{mg} / \mathrm{kg})$ followed by ear vein injection of propofol $(1.0 \mathrm{mg} / \mathrm{kg})$. Pentobarbital $(3 \%)$ was intravenously infused $(8 \mathrm{mg} / \mathrm{kg} / \mathrm{h})$ to maintain anesthesia. Subsequently, all animals were endotracheally intubated using a ventilator (Evita 4; Draeger Medical, Lübeck, Germany). The ventilation mode was synchronized to a tidal volume of $8 \mathrm{ml} / \mathrm{kg}$ and a respiratory frequency of 12-20 breaths per minute on room air. An inline infrared cacographic (Respirometric Inc., Murrysville, PA, USA) was maintained between 30 and $40 \mathrm{mmHg}$ to monitor the end-tidal pressure of $\mathrm{CO}_{2}$. An arterial catheter was inserted into the aortic arch from the left femoral artery for continuous measurement of the aortic pressure. An A7-Fr Swan-Ganz catheter (Edwards Life Sciences, Irvine, CA, USA) was placed into the pulmonary artery from the right external jugular vein to monitor the mean pulmonary artery pressure. Electrocardiograms and hemodynamics were also monitored (M8001A; Philips Medizin Systeme GmbH, Boeblingen, Germany). A triple lumen central venous catheter was placed in the left femoral vein to monitor the central venous pressure. During the procedures, normal saline was infused through the right femoral vein at $8 \mathrm{ml} / \mathrm{kg} / \mathrm{h}$ to compensate for fluid loss, as well as maintain a steady central venous pressure $(5-12 \mathrm{mmHg})$. A large-bore catheter (internal diameter, $1.0 \mathrm{~cm}$ ) was inserted from the left external jugular vein into the opening of the pulmonary artery by computerized tomography guidance for infusion of blood clots (9). All wounds were partially sutured.
Experimental protocol. Blood $(\sim 100 \mathrm{ml})$ was collected from the left femoral vein and allowed to self-coagulate at room temperature (2-3 h) as previously described (8). The clots $(10-15 \mathrm{ml})$ were cut into pieces $(1.5 \times 1 \times 1 \mathrm{~cm})$ and suspended in normal saline in a large catheter tip syringe $(1.0-\mathrm{cm}$ internal diameter, $50 \mathrm{ml}$ ). To obtain a model of APE-induced CA, the suspension of blood clots was injected through the left external jugular vein over 2 min until a mean arterial pressure (MAP) of $<30 \mathrm{mmHg}$ was reached (14). The model of massive APE was prepared successfully and diagnosed by chest-enhanced CT scanning. Subsequently, urokinase $(15,000 \mathrm{U} / \mathrm{kg}$, Nanjing Nanda Pharmaceutical Co., Ltd. Nanjing, China) was infused into the pulmonary artery using a Swan-Ganz catheter, and cardiopulmonary resuscitation (CPR) was initiated immediately according to the American Heart Association guidelines for CPR and emergency cardiovascular care from 2010 (15). Return of the spontaneous circulation (ROSC) was verified by a systolic blood pressure of $>50 \mathrm{mmHg}$ maintained for 10 consecutive minutes (16). Animals were considered dead with the absence of heart beat/breathing and eye reflex, and presence of rigor mortis if ROSC was not restored within $30 \mathrm{~min}$.

A total of 29 pigs were randomly assigned to three groups. Group 1 (control group; $n=5$ ) was injected with a bolus of propofol $(100 \mathrm{mg})$ followed by $10 \mathrm{ml}$ potassium chloride $(15 \%)$ at $6 \mathrm{~h}$ after initiation of the experiment. All animals in this group died due to ventricular fibrillation. Group 2 (APE-CA group; $n=5$ ) received a thrombus injection until the MAP reached $<30 \mathrm{mmHg}$, followed by CPR. All animals in this group died due to electromechanical diastolic arrest or ventricular fibrillation. Group 3 (APE-ROSC group; $\mathrm{n}=19$ ) received a thrombus injection until the MAP reached $<30 \mathrm{mmHg}$, followed by urokinase treatment and cardiopulmonary resuscitation, which was performed in accordance with the 2010 American Heart Association guidelines for cardiopulmonary resuscitation and emergency cardiovascular care (15). In this group, 10 animals were successfully resuscitated and randomly assigned to two subgroups: The ROSC-Cap group $(n=5)$ received an intravenous injection of captopril (22.22 mg/kg; cat. no. C8856-1G; Sigma-Aldrich, Merck KGaA, Darmstadt, Germany) at 30 min after ROSC, and the ROSC-SA group $(n=5)$ received the same volume of normal saline (SA) at $30 \mathrm{~min}$ after ROSC. Subsequently, all animals in the two groups were euthanatized using an intravenous injection of $10 \mathrm{ml}$ potassium chloride $(15 \%)$ following a bolus of propofol $(3.0 \mathrm{mg} / \mathrm{kg})$ at $6 \mathrm{~h}$ after ROSC. The cardiac tissue was isolated, immediately frozen in liquid nitrogen, and stored at $-80^{\circ} \mathrm{C}(17)$.

Histological evaluation. Myocardial tissue samples were fixed with $10 \%$ buffered formalin, embedded in paraffin, and stained using hematoxylin and eosin for further evaluation of pathological changes. Scoring of cardiac pathological changes was based on the criteria from the study of Rezkalla et al (18). In brief, five high-power visual fields were obtained from each slice, the ratio of the area of inflammatory cell infiltration and necrosis to the area of the whole visual field in each visual field was calculated. No lesion scored 0 points, lesion area $<25 \%$ scored 1 points, lesion area $25-49 \%$ scored 2 points, 50 to $75 \%$ of lesion area scored 3 points, lesion area $>75 \%$ scored 4 points (18). 
Western blot analysis. Using a radioimmunoprecipitation assay (RIPA) buffer and protease inhibitors (Roche, Basel, Switzerland), proteins from the left and right myocardium were extracted and isolated according to the manufacturer's protocol. Each tissue sample (20 mg) was chopped into fragments and homogenized in $200 \mu$ l RIPA buffer. Tissue lysates were centrifuged $\left(13,000 \times \mathrm{g}, 20 \mathrm{~min}, 4^{\circ} \mathrm{C}\right)$, and the supernatant was immediately collected and stored at $-80^{\circ} \mathrm{C}$ until further use. The protein concentration in the supernatants was measured using a Bicinchoninic Acid Protein Assay kit (Pierce; Thermo Fisher Scientific, Inc., Waltham, MA, USA). The centrifuged proteins were mixed with the same volume of 5X SDS buffer [2.5 ml 0.5 mol/1 Tris- $\mathrm{HCl}$ (pH 6.8), 0.5 g SDS, $0.39 \mathrm{~g}$ dithiothreitol, $2.5 \mathrm{ml}$ glycerol and $0.025 \mathrm{~g}$ bromophenol blue] to obtain a mixture at a final concentration of $3 \mu \mathrm{g} / \mu \mathrm{l}$. The homogenates were heated at $95^{\circ} \mathrm{C}$ for $5 \mathrm{~min}$. Total protein $(40 \mu \mathrm{g})$ was resolved by 8,10 and $15 \%$ SDS-PAGE using a standard biotinylated molecular weight marker (Biomed, Beijing, China). The samples were wet-transferred to a polyvinylidene difluoride membrane $(0.45 \mu \mathrm{m}$; EMD Millipore, Billerica, MA, USA) and stained using Ponceau-S Stain to assess equal loading of protein. The blots were blocked using 5\% non-fat dry milk for $2 \mathrm{~h}$ in a Tris-buffered saline solution with Tween-20 [TBST; 1 mol/1 Tris-HCl ( $\mathrm{pH} 7.5)$, $100 \mathrm{mM} \mathrm{NaCl}$ and 20\% Tween-20] and incubated overnight at $4^{\circ} \mathrm{C}$ with a mixture of ACE2 (cat. no. SC-390851), ACE (cat. no. SC-2079), Ang II receptor type 1 (AT1; cat. no. SC-81671), B-cell lymphoma 2 (Bcl-2)-associated X protein (Bax; cat. no. SC-6236) and Bcl-2 antibodies (1:500 dilution; cat. no. SC-492, Santa Cruz Biotechnology, Dallas, TX, USA), Mas receptor antibody (1:200 dilution; cat. no. AAR-013, Alomone Labs, Jerusalem, Israel), 1:1,000 dilution of cleaved caspase-3 (cat. no. 9664, Cell Signaling Technology, Inc., Danvers, MA, USA) and caspase-3 antibodies (cat. no. SC-7272, Santa Cruz Biotechnology, Inc.) and $\beta$-actin antibody (1:1,000 dilution; cat. no. ab8226, Abcam, Cambridge, MA, USA). After washing with TBST, the blots were incubated with horseradish peroxidase-conjugated anti-rabbit or anti-mouse immunoglobulin G (1:10,000; cat. no. 111-035-003; Jackson ImmunoResearch Laboratories, Inc., West Grove, PA, USA) as the secondary antibody for $40 \mathrm{~min}$ at room temperature. After washing the blots with TBST again, the bands were visualized using enhanced Immobilon ${ }^{\mathrm{TM}}$ Western chemiluminescent HRP substrate (cat. no. WBKLSO500, Millipore, Billerica, MA, USA) and analyzed using a Gel Image System version 4.00 (Tanon Science \& Technology Co., Ltd., Shanghai, China) was used for densitometric quantification of the blots.

Immunohistochemistry. Myocardial tissues were fixed in $10 \%$ buffered formalin for $48 \mathrm{~h}$ at room temperature, dehydrated in a sequence of $100,95,70$ and $50 \%$ graded alcohol solutions, sliced at $4 \mu \mathrm{m}$ thickness, and embedded in paraffin. The sections were rinsed in histosol, rehydrated in a graded series of ethanols and blocked with $5 \%$ bovine serum albumin (Sigma-Aldrich; Merck KGaA) for $4 \mathrm{~h}$. Sections were incubated with anti-Mas receptor antibody (1:200 dilution; cat. no. AAR-013, Alomone Labs, Jerusalem, Israel) and anti-phosphorylated (p)-ERK1/2 antibody (1:1,000 dilution; cat. no. $4376 \mathrm{~S}$, Cell Signaling Technology, Inc.) at $4^{\circ} \mathrm{C}$ overnight. After rinsing with PBS, sections were incubated in polyperoxidase-conjugated secondary antibody (1:200 dilution; PV-9001, ZSGB-Biotechnology, Beijing, China) for $2 \mathrm{~h}$ at room temperature. Diaminobenzidine tetrahydrochloride (Sigma-Aldrich; Merck KGaA) was then used to stain the sections. After counterstaining with hematoxylin, sections were viewed under an inverted phase microscope (IX80 microscope, Olympus Corporation, Tokyo, Japan). Image-Pro Insight 6.0 (Media Cybernetics, Rockville, MD, USA) was used for image analysis.

Ultrastructural analysis. Electron microscopy was employed to evaluate ultrastructural markers of myocardial cell apoptosis. Samples of the left and right myocardium were collected from pigs within 1-2 min of being euthanized. The myocardium was sliced $(1 \mathrm{~mm})$ on ice, fixed in $2.5 \%$ (v/v) glutaraldehyde at $4^{\circ} \mathrm{C}$ for $30 \mathrm{~min}$, post-fixed in $1 \%(\mathrm{v} / \mathrm{v})$ osmic acid for $1 \mathrm{~h}$, and dehydrated via a graded ethanol series and embedded in Epon 812 at $40^{\circ} \mathrm{C}$ for $4 \mathrm{~h}, 50^{\circ} \mathrm{C}$ for $2 \mathrm{~h}$ and $90^{\circ} \mathrm{C}$ for $12 \mathrm{~h}$. Thinner sections $(0.5-1 \mu \mathrm{m})$ were prepared, stained with $0.5 \%(\mathrm{w} / \mathrm{v})$ toluidine blue and viewed under a light microscope (Olympus). Ultra-thin sections $(\sim 60 \mathrm{~nm})$ were cut, double-stained with $2.0 \%$ (w/v) uranyl acetate for $20 \mathrm{~min}$ and $2.0 \%(\mathrm{w} / \mathrm{v})$ lead citrate for $15 \mathrm{~min}$, and viewed under a transmission electron microscope (Hitachi HT7700; Hitachi Ltd., Tokyo, Japan).

Statistical analysis. Values are expressed as the mean \pm standard deviation. SPSS 16.0 (SPSS, Inc., Chicago, IL) was used for data analyses, and one-way analysis of variance coupled with Bonferroni's correction (Newman-Keuls test) was used for post-hoc comparisons. Continuous variables were assessed for normal distribution by using the Kolmogorov-Smirnov test and equal variances by the homogeneity of variance test. Pearson's correlation coefficient was calculated to determine the correlations between the ratios of ACE2/ACE, as well as between caspase-3, Bcl-2, Bax and Bcl-2/Bax. $\mathrm{P}<0.05$ was considered to indicate a statistically significant difference.

\section{Results}

Histological analysis of the myocardium following APE-CA. Histological analyses of the left and right ventricular tissues isolated following APE-CA and ROSC were performed. The control group exhibited no lesions in the myocardial cells and the myocardial fibers were normal (Fig. 1). In contrast to the control group, the APE-CA and ROSC groups displayed myocardial fiber dissolution or fracture in the central zones of the left and right ventricles, which significantly increased the pathological score (all $\mathrm{P}<0.05)$. Captopril partially inhibited this effect (Fig. 1). Electron microscopy analysis of myocardial tissues in the APE-CA, ROSC-SA and ROSC-Cap groups revealed nuclear swelling, as well as myofilament dissolution and intercalated disks broken or dissolved (Fig. 2; black arrows), and mitochondrial engorgement and myocardial cell apoptosis (Fig. 2; red arrows). The increase in apoptosis in the left myocardium was less pronounced in the captopril-treated group (Fig. 2).

Imbalance of the ACE2/ACE axis in left and right myocardium following APE-CA. As presented in Fig. 3A and B, in 


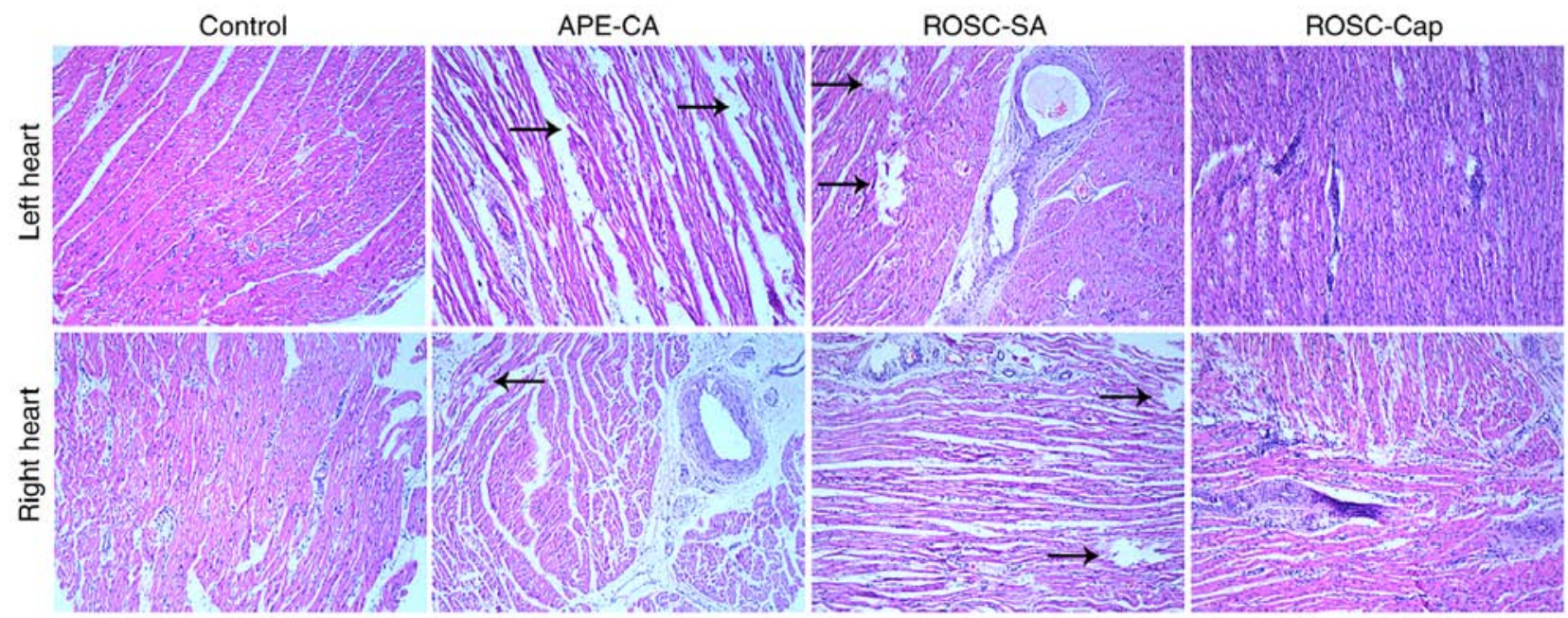

Figure 1. Histological analysis following APE-CA and ROSC and the effects of captopril. Representative images of H\&E-stained myocardial tissues (magnification, x100). Black arrows indicate fractured myocardial fibers. APE-CA, acute pulmonary embolism with cardiac arrest; ROSC, return of the spontaneous circulation; Cap, captopril; SA, saline.
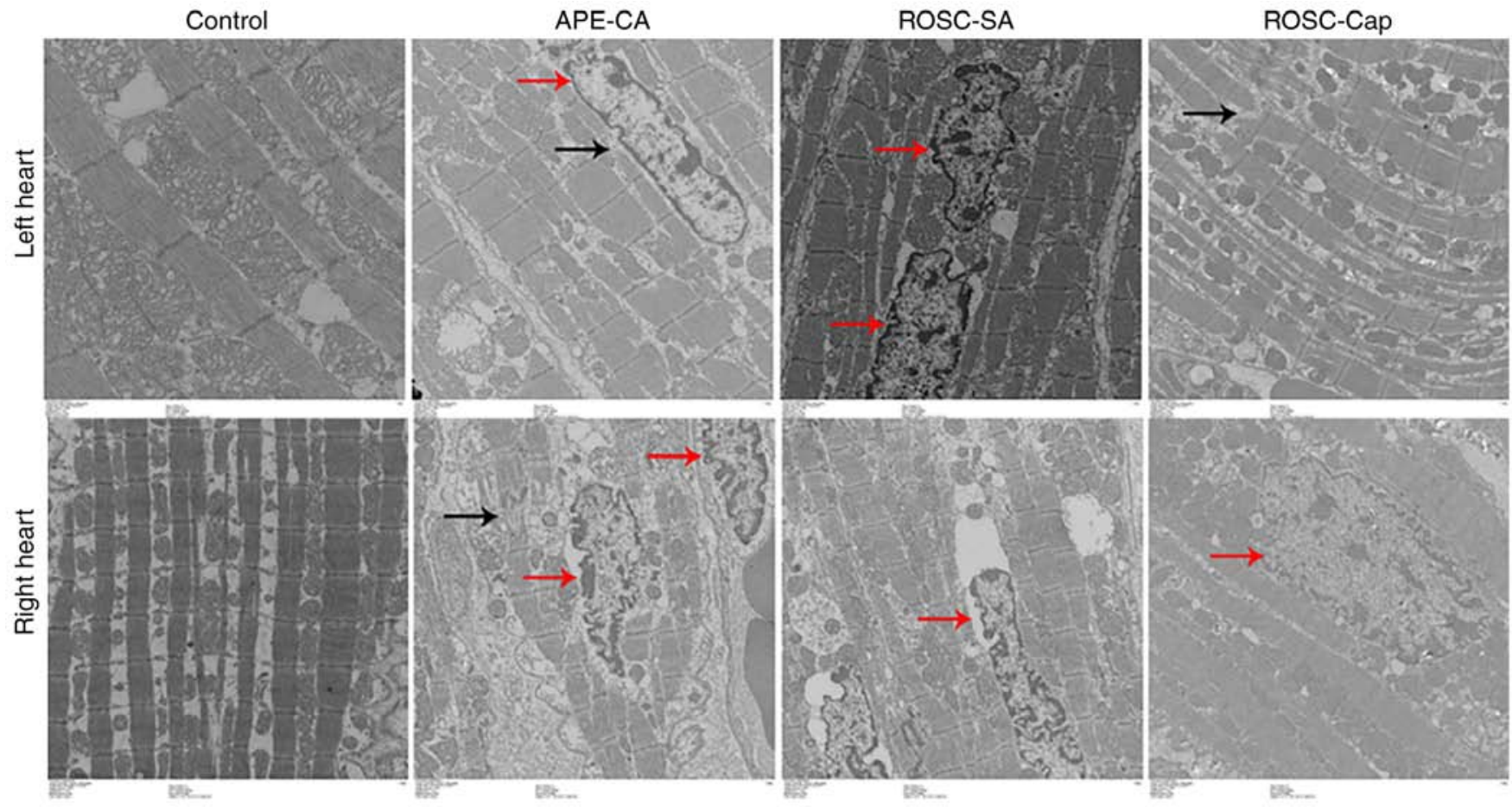

Figure 2. Cytoplasmic ultrastructure of the myocardium viewed under a transmission electron microscope at high magnification (x20,000). Black arrows indicate myocardial fibers and the junction between myocardial cells called intercalated discs that are broken or dissolved. Red arrows indicate apoptotic cells. Treatment with captopril decreased the number of apoptotic cells in the left myocardium. APE-CA, acute pulmonary embolism with cardiac arrest; ROSC, return of the spontaneous circulation; Cap, captopril; SA, saline.

the left myocardium, the expression levels of ACE $(\mathrm{P}<0.001$ and $\mathrm{P}<0.01$, respectively), the AT1 receptor $(\mathrm{P}<0.001$ and $\mathrm{P}<0.01$, respectively) and the Mas receptor $(\mathrm{P}<0.001$ for each) in the APE-CA and ROSC groups were significantly higher compared with those in the control group. Decreases in ACE and AT1 receptor expression were observed in the ROSC-SA vs. those in the APE-CA group $(\mathrm{P}<0.05$ and $\mathrm{P}<0.01$, respectively). However, the levels of ACE2 and Mas receptor were not significantly different between the ROSC-SA and APE-CA groups. Immunohistochemical analysis of the left myocardium revealed positive staining for the Mas receptor, primarily in the cell nuclei, of the control group, while staining was evident in the cell nuclei and cytoplasm of the APE-CA and ROSC-SA groups (Fig. 3C). A marked increase in Mas receptor expression was observed in the APE-CA and ROSC groups compared with that in the control group ( $\mathrm{P}<0.05$ for each; Fig. $3 \mathrm{C})$. No changes in the proteins of the ACE2 axis and ACE axis were observed in the right myocardium following either APE-CA or ROSC (Fig. 3D and E). 
A

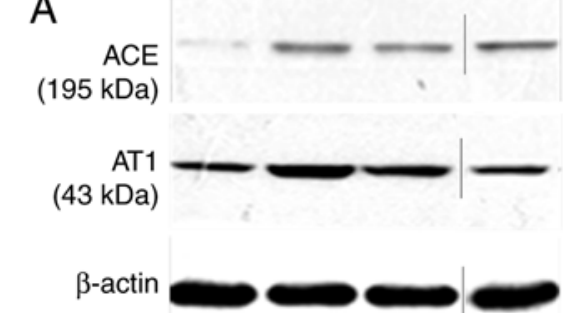

B

Control APE-CA ROSC-SA ROSC-Cap
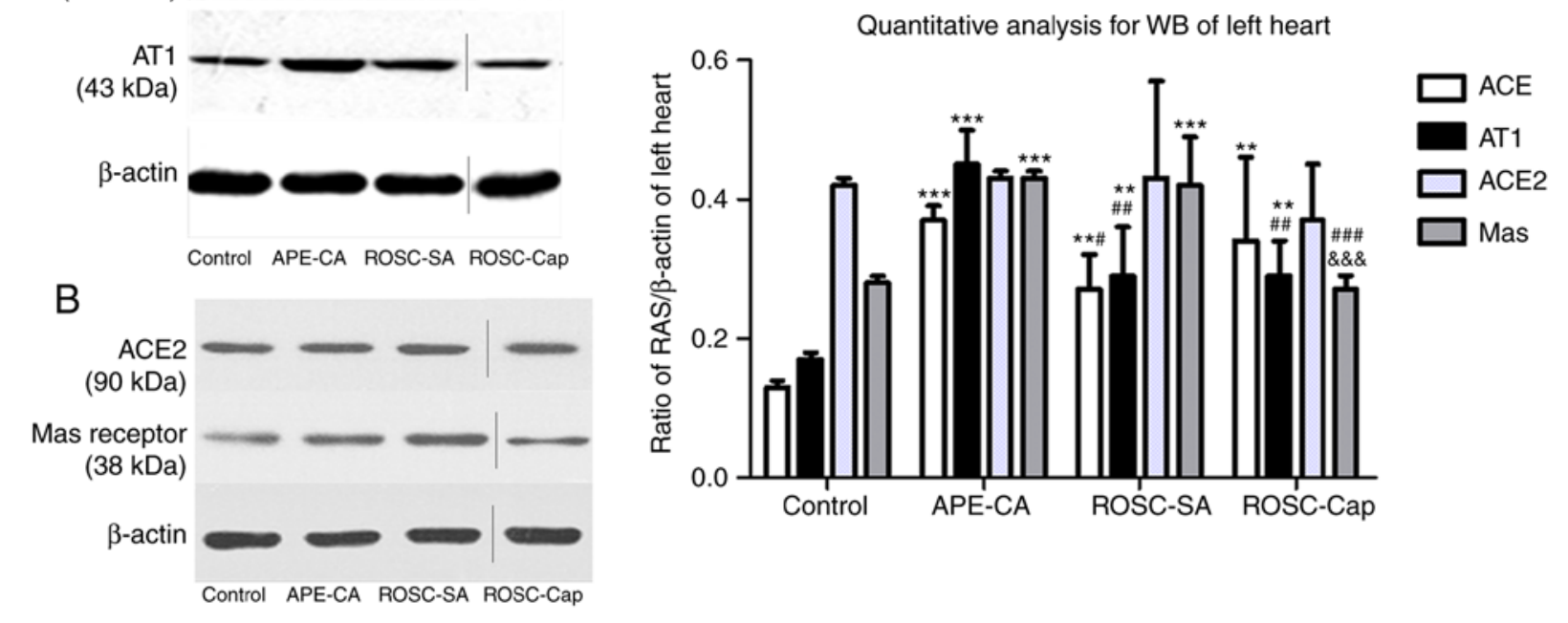

C
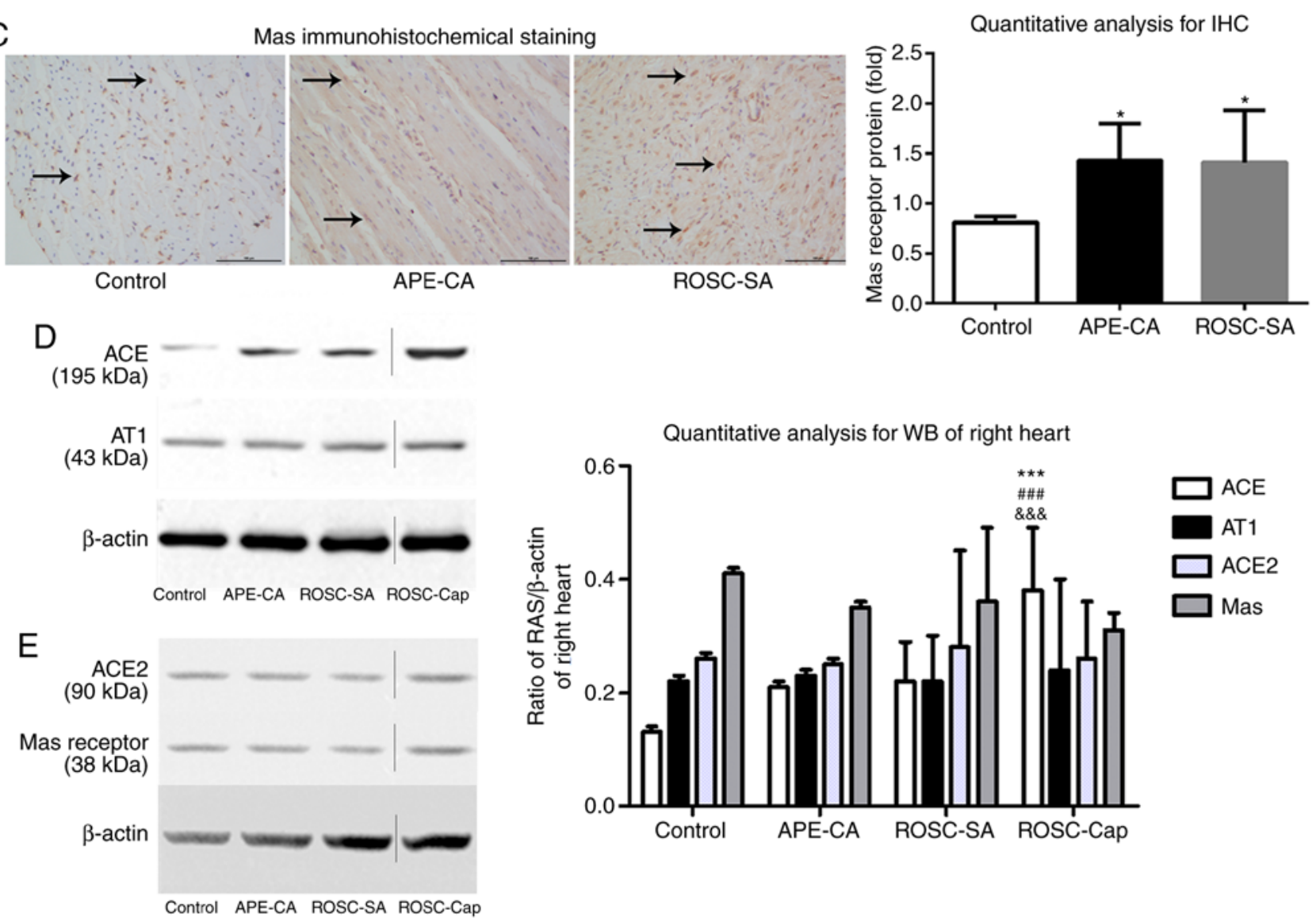

Figure 3. Expression of proteins of the RAS in the left myocardium as determined by (A and B) WB analysis and (C) immunohistochemistry (scale bar, $100 \mu \mathrm{m}$; black arrows indicate expression of the Mas receptor). (D and E) Expression of members of the RAS in the right myocardium as determined by WB analysis. Vertical lines in the western blot indicate non-adjacent bands from the same gel. Values are expressed as the mean \pm standard deviation, $\mathrm{N}=5$ pigs per group. ${ }^{*} \mathrm{P}<0.05 ;{ }^{* *} \mathrm{P}<0.01 ;{ }^{* * *} \mathrm{P}<0.001$ vs. control group; ${ }^{\# \mathrm{P}}<0.05$; ${ }^{\# \#} \mathrm{P}<0.01$; ${ }^{\# \# \#} \mathrm{P}<0.001$ vs. the APE-CA group; \&\&\& $\mathrm{P}<0.001$ vs. the ROSC-SA group; APE-CA, acute pulmonary embolism with cardiac arrest; ROSC, return of the spontaneous circulation; SA, saline; ACE, angiotensin-converting enzyme; RAS, renin-angiotensin system; IHC, immunohistochemistry; AT1, angiotensin II receptor type 1; WB, western blot.

Differences in proteins of the RAS between left and right myocardium. The present study indicated a significant increase in the levels of ACE, AT1 and Mas in left myocardial tissues compared with those in right myocardial tissues across the experimental groups (Fig. 4A-C). In the ROSC-SA group, the differences in ACE and AT1 expression between the left and right myocardium were significantly decreased compared with those in the APE-CA group $(\mathrm{P}<0.001$ and $\mathrm{P}<0.05$; Fig. $4 \mathrm{~A}$ and $\mathrm{B}$ ). The difference in the ACE2/ACE ratio between the left and right myocardium was reduced in 
A

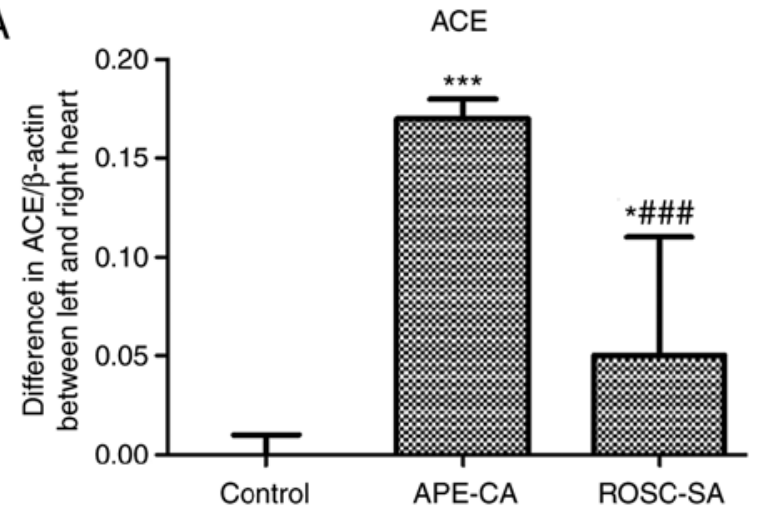

C

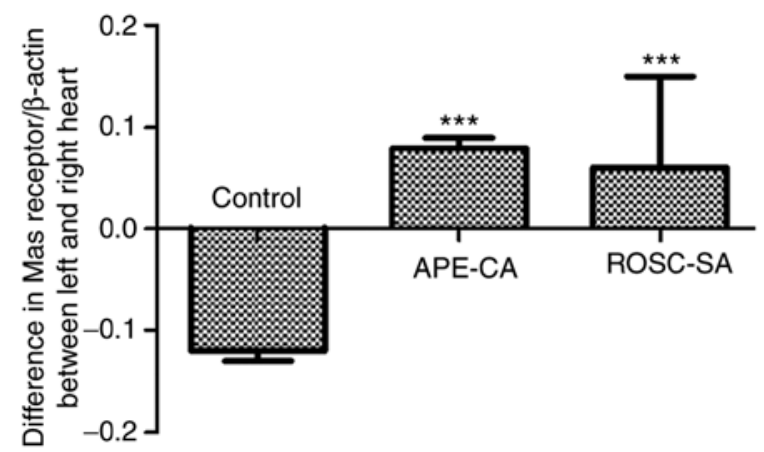

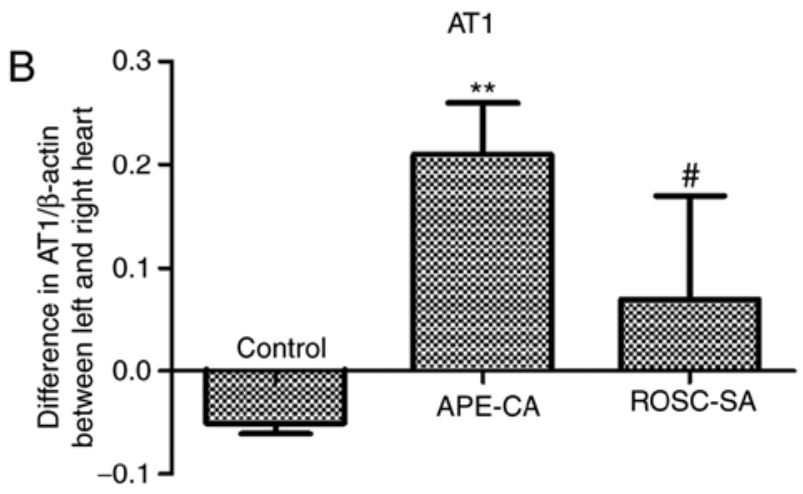

ACE2/ACE

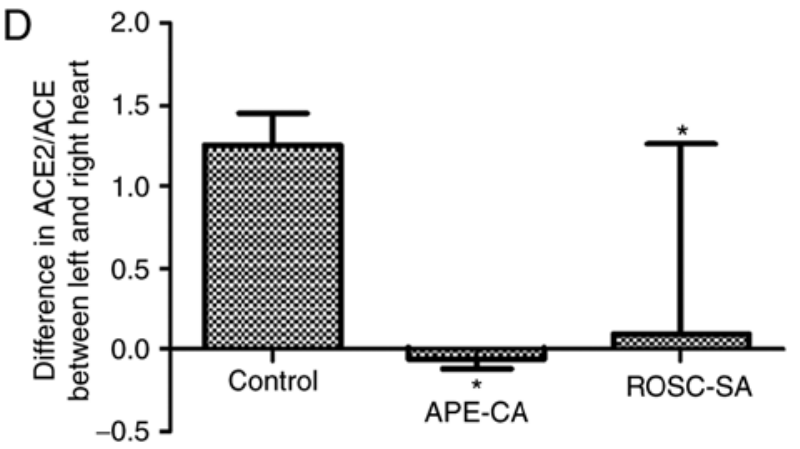

Figure 4. Differences in expression of proteins of the renin-angiotensin system between the left and right myocardium in APE-CA. (A) ACE, (B) AT1 receptor, (C) Mas receptor and (D) ACE2/ACE ratio. N=5 pigs per group. ${ }^{*} \mathrm{P}<0.05 ;{ }^{* *} \mathrm{P}<0.01 ;{ }^{* * *} \mathrm{P}<0.001$ vs. the control group; ${ }^{\#} \mathrm{P}<0.05 ;{ }^{\# \# \#} \mathrm{P}<0.001$ vs. the APE-CA group. APE-CA, acute pulmonary embolism with cardiac arrest; ROSC, return of the spontaneous circulation; SA, saline; ACE, angiotensin-converting enzyme; AT1, angiotensin II receptor type 1.

the APE-CA and ROSC-SA groups compared with that in the control group $(\mathrm{P}<0.05$; Fig. 4D). This effect was likely due to the activation of ACE expression in the left myocardium and ACE2 expression in the right myocardium.

Effects of captopril on apoptotic signaling following APE-CA. To elucidate the mechanism of action of captopril to inhibit myocardial apoptosis, the levels of the apoptosis-associated proteins Bax and Bcl-2 in the myocardium were assessed. In the left myocardium, Bax expression was increased $(\mathrm{P}<0.001$ and $\mathrm{P}<0.05$, respectively), while $\mathrm{Bcl}-2$ expression $(\mathrm{P}<0.001$ and $\mathrm{P}<0.05$, respectively) was decreased in the APE-CA and ROSC-SA groups compared with those in the control group (Fig. 5A). In the ROSC-SA group, inhibition of myocardial apoptosis was indicated by decreased Bax and increased Bcl-2 expression compared with that in the APE-CA group $(\mathrm{P}<0.01$ and $\mathrm{P}<0.05$, respectively; Fig. 5A). Compared with the saline group, after ROSC administration, Bax expression increased, whereas Bcl-2 expression decreased following captopril treatment in the left myocardium $(\mathrm{P}<0.01$ and $\mathrm{P}<0.05$; Fig. $5 \mathrm{~A})$, confirming the anti-apoptotic effect of captopril in the left heart. In the right heart, Bax expression was significantly elevated in the APE-CA and ROSC-SA groups $(\mathrm{P}<0.05$ for each; Fig. 5B) compared with that in the control group.

Regarding the levels of caspase- 3 in the left heart, a significant increase in the APE-CA and ROSC-SA groups compared with the control group was noted $(\mathrm{P}<0.05$ and $\mathrm{P}<0.01$, respectively; Fig. 5A). In addition, captopril treatment after ROSC decreased the levels of caspase- 3 compared with those in the saline-treated group $(0.34 \pm 0.06$ vs. $0.19 \pm 0.07$, $\mathrm{P}<0.001$; Fig. 5A). In the right heart, the levels of cleaved caspase-3 (17/19 KD) were significantly increased in the ROSC-SA group compared with those in the control group $(\mathrm{P}<0.01$; Fig. 5B). However, captopril had no inhibitory effect on cleaved caspase-3 following ROSC (Fig. 5B). These results suggest that captopril inhibits apoptosis in the left ventricle but may be enhanced slightly in the right myocardium.

Correlation between ACE2/ACE axis imbalance and apoptotic signaling proteins in the left myocardium. In the present study, the ACE2/ACE ratio was positively correlated with the levels of Bcl-2 ( $\mathrm{r}=0.792, \mathrm{P}<0.01)$ and the $\mathrm{Bcl}-2 / \mathrm{Bax}$ ratio $(\mathrm{r}=0.884, \mathrm{P}<0.001)$, and negatively correlated with the levels $\operatorname{Bax}(r=-0.844, \mathrm{P}<0.01)$ and caspase $-3(\mathrm{r}=-0.789, \mathrm{P}<0.01)$ in the control, APE-CA and ROSC-SA groups (Fig. 6A). These results support the hypothesis that an imbalance in the ACE2/ACE axis affects apoptosis of the left myocardium during APE-CA.

ERK1/2 activation in the myocardium and effects of captopril during ROSC following APE-CA. To study the molecular mechanism of action of captopril in the myocardium, immunohistochemical analysis of p-ERK1/2 in the left myocardium was performed. The images indicated that $\mathrm{p}$-ERK1/2-positive cells were located primarily in the nuclei of cardiomyocytes in the control group, but in the nuclei and cytoplasm of the 

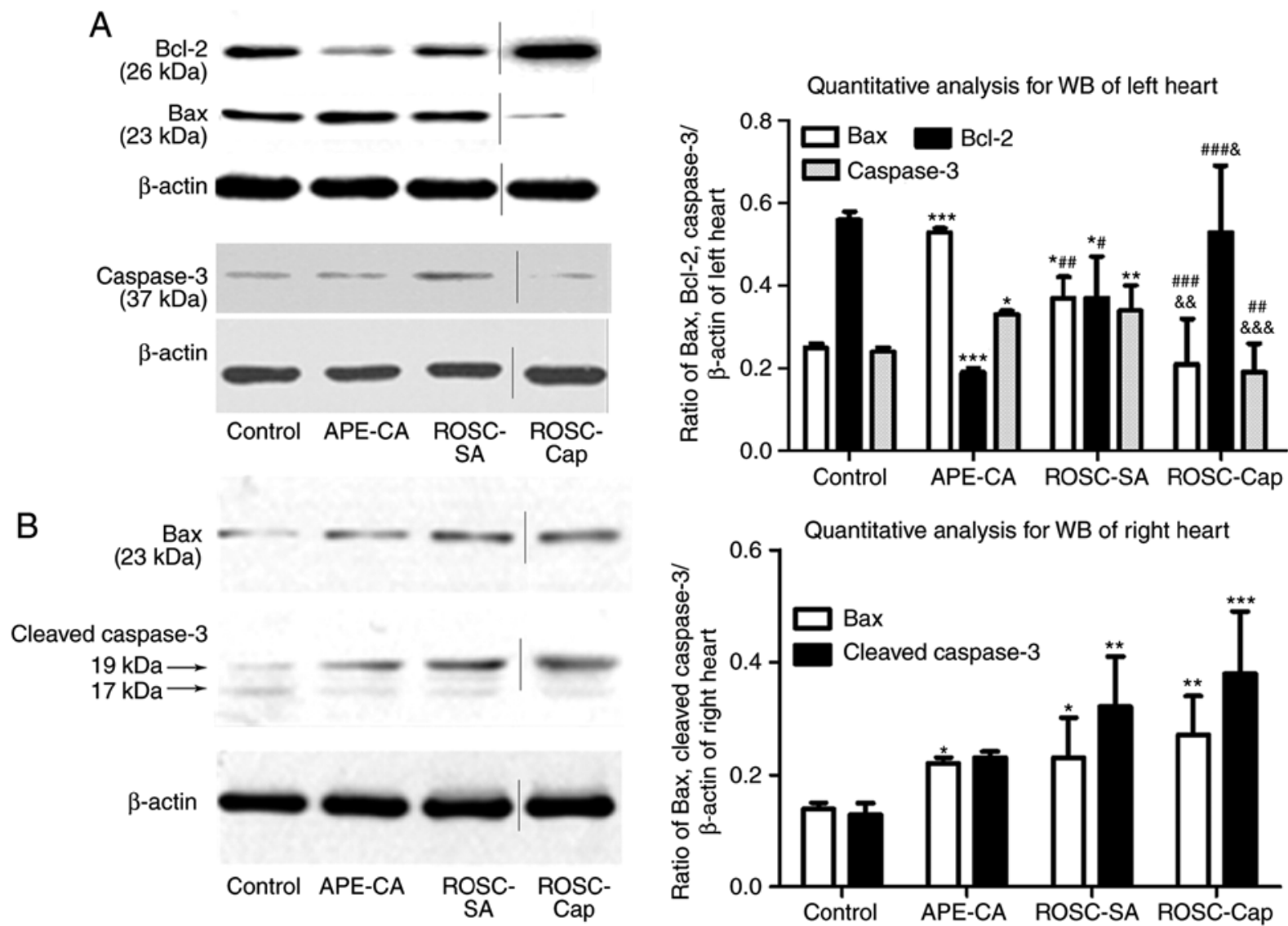

Figure 5. Expression of apoptotic signaling proteins in the left and right heart as determined by WB. (A) Representative images and quantitative analysis of Bax, Bcl-2 and caspase-3 in the left heart. (B) Representative images and quantitative analysis of Bax and cleaved caspase-3 in the right heart. Vertical lines in the western blot indicate non-adjacent bands from the same gel. $\mathrm{N}=5$ pigs per group. ${ }^{*} \mathrm{P}<0.05 ;{ }^{* * *} \mathrm{P}<0.01 ;{ }^{* * *} \mathrm{P}<0.001$ vs. the control group; ${ }^{\#} \mathrm{P}<0.05 ;{ }^{\# \#} \mathrm{P}<0.01$;

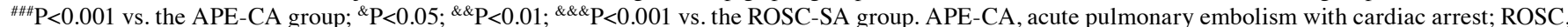
return of the spontaneous circulation; SA, saline; WB, western blot; Cap, captopril; Bcl-2, B-cell lymphoma 2; Bax, Bcl-2-associated X.

APE-CA and ROSC groups (Fig. 6B). Compared to the control group, a 5.35-fold and 4.27-fold increase in p-ERK1/2 was determined in the APE-CA and ROSC-SA group, respectively. Of note, treatment with captopril reduced this level by $26.57 \%$ in the ROSC-Cap group compared with that in the ROSC-SA group (Fig. 6C). In the right myocardium, no significant differences in the p-ERK1/2 levels were observed among the four groups (data not shown).

\section{Discussion}

The present study provides important insight regarding myocardial apoptosis following APE induced CA and ROSC. First, it was demonstrated that APE-CA induces myocardial apoptosis and myocardial fiber fracture. Furthermore, an imbalance in the ACE2/ACE axis was revealed to be a consequence of differential activation of the ACE axis in the left myocardium and the ACE2 axis in the right myocardium following APE-CA and ROSC as shown in Fig. 4. Finally, the results revealed that captopril reduces left myocardial injury and apoptosis, as evidenced by increased Bcl-2 expression and decreased Bax and caspase-3 expression. However, there were some marked increases in apoptosis in the right myocardium associated with captopril, but they were not significant.
The major pathophysiological features of APE include endogenous or exogenous embolus, pulmonary hypertension and acute right ventricular dilation, ventricular interdependence, lower left ventricular diastolic compliance, acute cardiogenic shock and even death (19). Thus, massive APE with CA results in an imbalance of right and left ventricular function. Kumamaru et al (20) determined that a normal right-to-left ventricular ratio of $<0.97$ was sufficient to exclude right ventricular strain/pulmonary embolism-associated short-term death. The present study aimed to further clarify how pathophysiological mechanisms of APE differ between the left and right heart.

The RAS maintains cardiovascular stability, specifically via the classic ACE/Ang II/AT1 receptor axis and a recently identified ACE2/Ang(1-7)/Mas receptor axis. Thus, the duality of the RAS (its ability to stimulate apoptosis, vasoconstriction, proliferation and fibrosis, while also being able to initiate anti-apoptotic, vasodilatory, anti-proliferative and anti-fibrotic pathways) is mainly driven by a balanced ACE2/ACE axis (21). The present study demonstrates that the RAS is activated during a massive APE when the ACE2/ACE ratio is decreased in the left, but not in the right myocardium. This observation may be explained by the fact that, during an APE, the blood supply from the coronary artery to the left heart is significantly reduced and the left ventricular pressure and coronary perfusion pressure exhibit marked decreases, leading to activation of the RAS. 


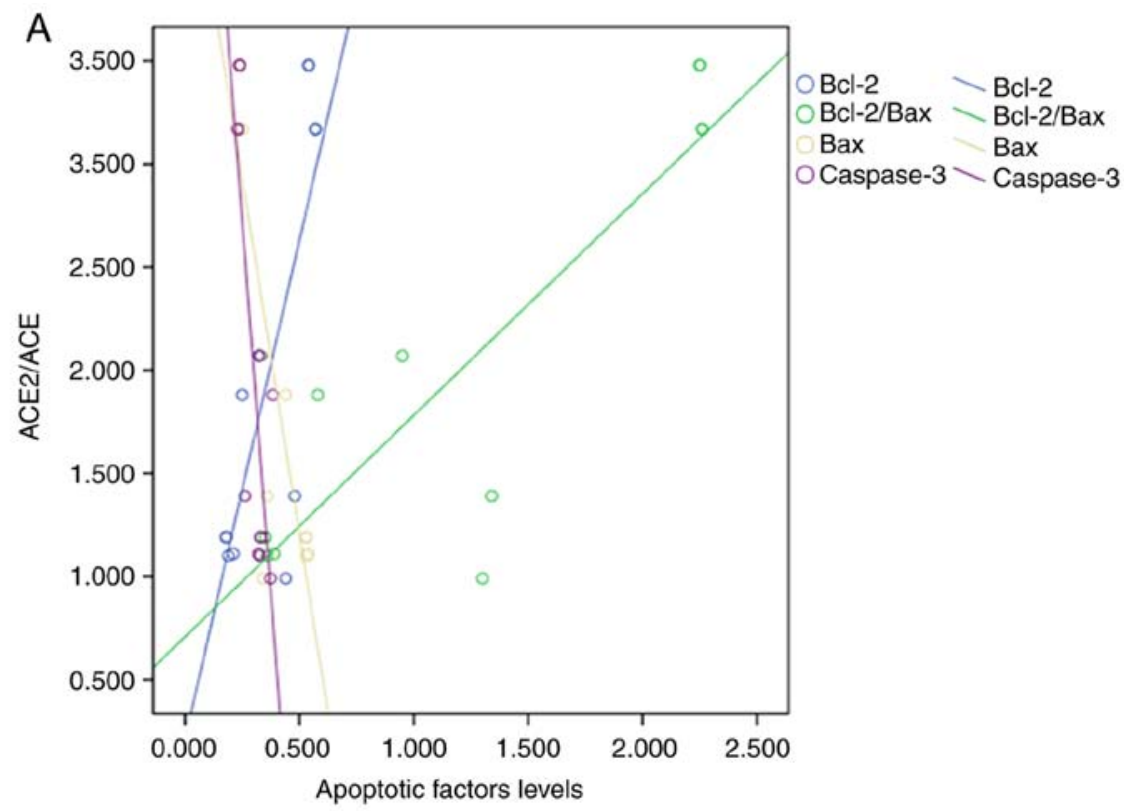

B

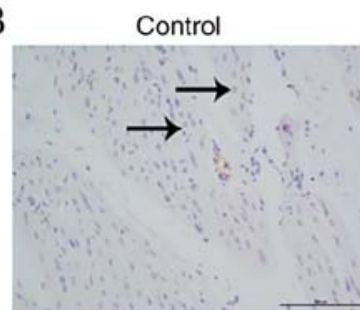

ROSC-SA

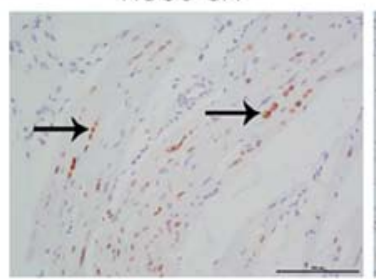

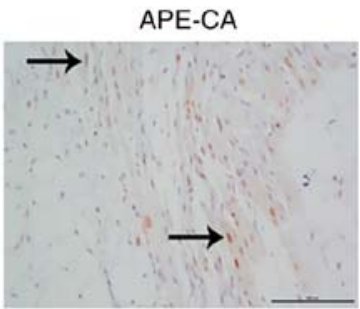

ROSC-Cap

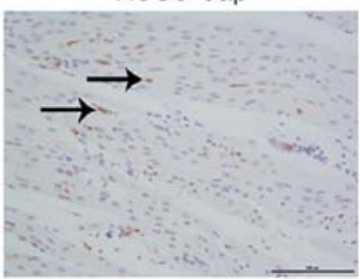

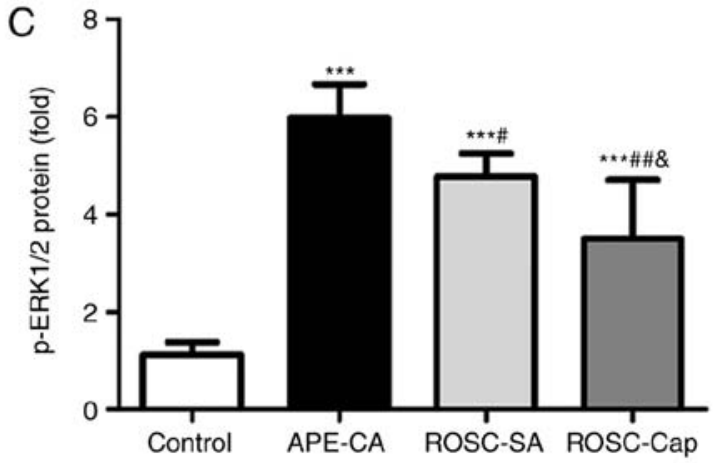

Figure 6. ACE2/ACE axis imbalance and apoptotic signaling proteins, and p-ERK1/2 in the left myocardium of APE-CA and ROSC pigs as determined by immunohistochemistry. (A) Correlation between the ACE2/ACE axis and apoptosis-associated proteins. (B) Representative images. Black arrows indicate staining for p-ERK1/2 (scale bar, $100 \mu \mathrm{m}$ ). (C) Quantitative analysis of immunohistochemical staining. $\mathrm{N}=5$ pigs per group. ${ }^{* *} \mathrm{P}<0.01 ;{ }^{* * *} \mathrm{P}<0.001 \mathrm{vs}$. the control group; ${ }^{\#} \mathrm{P}<0.05 ;{ }^{\# \#} \mathrm{P}<0.01$ vs. the APE-CA group; ${ }^{\&} \mathrm{P}<0.05$ vs. the ROSC-SA group. APE-CA, acute pulmonary embolism with cardiac arrest; ROSC, return of the spontaneous circulation; SA, saline; Cap, captopril; p-ERK, phosphorylated extracellular signal-regulated kinase.

Zagorski et al (22) reported that the mortality rate due to severe pulmonary embolism in rats was $37.8 \%$, which is similar to the mortality rate of $47.4 \%$ in the model of the present study. Schultz et al (23) employed an in vivo APE model in pigs and mentioned that 'a stable model of high-risk PE in pigs with an acceptable variance and mortality rate may therefore be difficult to develop.' This suggests that the mortality rates in this model are expected to be high. This would justify the high percentage of mortality observed in the present study. Of note, a large proportion of APE patients die of heart failure following ROSC (4). A study including 778 comatose survivors of CA reported that $65 \%$ of patients died within 1 week after resuscitation, and $>50 \%$ of the deaths were attributed to heart failure $(24,25)$. Previous studies confirm that myocardial apoptosis has a major role in cardiac dysfunction $(5,26)$. When a myocardial cell is stimulated by certain pathological or physiological factors, the intracellular mitochondrial channel releases macromolecules, including cytochrome $c$ (Cyt-C), pro-caspase-2 and pro-caspase-9. Cleavage of these released macromolecules by proteases activates apoptotic peptidase activating factor- 1 and apoptosis factor- 1 to form a complex that subsequently activates caspase-9 and caspase-3. Bcl-2 regulates the mitochondrial membrane permeability, the release of the abovementioned pro-apoptotic factors from the mitochondria and mitochondrial apoptosis. Constitutive expression of $\mathrm{Bcl}-2$ attenuates Bax-mediated Cyt-C release, and inhibits caspase activation and transfer of apoptosis-inducing proteins from the mitochondria to the nucleus (27). Recently, it was demonstrated that certain ribosomal proteins (rp), including rpL3, are released from the nucleolus and activate mitochondrial apoptotic pathways by increasing the Bax/Bcl-2 ratio (28).

In the present APE-CA model, an increased Bax expression in the left and right myocardium, increased caspase-3 activation and downregulation of Bcl-2 in the left myocardium were observed. Furthermore, in captopril-treated pigs, the 
expression of Bcl-2 in the left myocardium was increased and that of Bax and caspase-3 was decreased compared with that in SA-treated pigs, confirming the anti-apoptotic effect of captopril only on the left heart following ROSC.

The present results are consistent with those of previous studies indicating that the classic ACE/AngII/AT1 receptor axis promotes myocardial apoptosis, while the novel ACE2/Ang(1-7)/Mas receptor axis inhibits apoptosis. Liu et al (7) demonstrated that in H9c2 cells, AngII increases the apoptotic index four-fold of that in untreated control cells $(\mathrm{P}<0.01)$. Fabris et al (29), reported that the ACE inhibitor ramipril is effective in preventing ovariectomy-induced cardiac apoptosis associated with reduced cardiac ACE and AT1 receptor gene expression. Another previous study demonstrated that in endothelial cells isolated from the coronary arteries of dogs following cardiopulmonary bypass, captopril markedly inhibited hemoglobin-based oxygen carrier-induced apoptosis by reducing associated decreases in the Bcl-2/Bax ratio and increases in caspase-3 cleavage (30). Qi et al (8) reported that in a model of myocardial ischemia induced by coronary artery ligation surgery, ACE2 overexpression significantly preserved cardiac function and reduced the infarct size, presumably by inhibiting cell apoptosis. Furthermore, Ang-(1-7) treatment significantly protected H9C2 rat cardiomyocytes from hypoxia/reoxygenation-induced oxidative injury by reducing cell apoptosis (31). Wan et al (32) indicated that genetic silencing of intracellular ACE markedly stimulated ACE2 expression, caused a downregulation of caspase-3 and Bax expression, and promoted Bcl-2 expression in $\mathrm{H} 9 \mathrm{c} 2$ cardiomyocytes in response to anoxia/reoxygenation.

It has been proposed that ERK activation is associated with the progression of heart failure. Increased expression of p-ERK has been reported in pressure overload-induced heart failure (33). The association of ERK1/2 with lipopolysaccharide-induced $\mathrm{H} 9 \mathrm{c} 2$ cell apoptosis has also been reported (34). Lei et al (11) recently demonstrated that the increase of p-ERK1/2 in high glucose-induced H9c2 cells may be significantly suppressed by treatment with Ang-(1-7). In addition, irbesartan or ramipril treatment prevented high salt diet-induced left ventricular hypertrophy and lowered ERK phosphorylation (12). It was also confirmed that a loss of ACE2 leads to increased p-ERK1/2 in myocardial infarct and peri-infarct regions (35). The observations of the present study support the collective results of the aforementioned studies, in terms of inhibition of left cardiac ERK1/2 activation by captopril treatment corresponding to a lower incidence of myocardial apoptosis.

Of note, the present study has several limitations. First, the sample size was relatively small and the statistical power may have been insufficient to detect additional inter-group differences. The present study focused on the roles of imbalances in the ACE2/ACE axis, not on the ACE2 axis alone, in myocardial apoptosis in massive APE. Thus, future studies addressing the direct involvement of the ACE2 axis in massive APE should be designed using recombinant ACE2 or ACE2 gene knockout models.

In conclusion, the present study indicated that an imbalance in the ACE2/ACE axis is associated with apoptosis following massive APE and demonstrated the protective effects of post-resuscitation captopril against myocardial apoptosis.

\section{Acknowledgements}

The authors would like to thank Miss Nathalie in Medjaden Bioscience, Ltd. (Hong Kong, China) for assisting with the preparation of this manuscript

\section{Funding}

The present study was supported by the National Natural Science Foundation of China (grant no. 81372025), the 2015 Annual Special Cultivation and Development Project for Technology Innovation Base of Beijing Key Laboratory of Cardiopulmonary Cerebral Resuscitation (grant no. Z151100001615056), the Natural Science Foundation of Beijing Municipality (grant no. 7173253) and the Beijing Municipal Administration of Hospitals' Youth Programme (grant no. QML20170105). The funders had no role in the study design, data collection and analysis, writing of the manuscript or decision to publish.

\section{Availability of data and materials}

The datasets used and/or analyzed during the current study are available from the corresponding author on reasonable request.

\section{Authors' contributions}

CSL, MRX and HLX designed the study; HLX, LXZ, JY, NT and LA performed the experiments and analyzed the data; QTL provided chest CT scans; HLX wrote the manuscript. All authors approved the final version for publication.

\section{Ethics approval and consent to participate}

The Committee on the Ethics of Animal Experiments of Capital Medical University approved the study procedures (permit no. 2010-D-013). Experimental protocols were designed in strict compliance with the Animal Care Guidelines of the Institutional Animal Care and Use Committee of Capital Medical University (Beijing, China) as well as the Utstein-style guidelines (13).

\section{Patient consent for publication}

Not applicable.

\section{Competing interests}

The authors declare that they have no competing interests regarding this study.

\section{References}

1. Dahhan T,Alenezi F,SamadZand Rajagopal S: Echocardiography in the risk assessment of acute pulmonary embolism. Semin Respir Crit Care Med 38: 18-28, 2017.

2. Jiménez D, Lobo JL, Barrios D, Prandoni P and Yusen RD: Risk stratification of patients with acute symptomatic pulmonary embolism. Intern Emerg Med 11: 11-18, 2016.

3. White RH: The epidemiology of venous thromboembolism. Circulation 107 (23 Suppl 1): I4-I8, 2003.

4. Yin Q, Li X and Li C: Thrombolysis after initially unsuccessful cardiopulmonary resuscitation in presumed pulmonary embolism. Am J Emerg Med 33: 132.e1-e2, 2015. 
5. Sabbah HN: Apoptotic cell death in heart failure. Cardiovasc Res 45: 704-712, 2000.

6. De Mello WC: Local renin angiotensin aldosterone systems and cardiovascular diseases. Med Clin North Am 101: 117-127, 2017.

7. Liu JJ, Li DL, Zhou J, Sun L, Zhao M, Kong SS, Wang YH, Yu XJ, Zhou J and Zang WJ: Acetylcholine prevents angiotensin II-induced oxidative stress and apoptosis in H9c2 cells. Apoptosis 16: 94-103, 2011.

8. Qi YF, Zhang J, Wang L, Shenoy V, Krause E, Oh SP, Pepine CJ, Katovich MJ and Raizada MK: Angiotensin-converting enzyme 2 inhibits high-mobility group box 1 and attenuates cardiac dysfunction post-myocardial ischemia. J Mol Med (Berl) 94: 37-49, 2016.

9. Xiao HL, Li CS, Zhao LX, Yang J, Tong N, An L and Liu QT: Captopril improves postresuscitation hemodynamics protective against pulmonary embolism by activating the ACE2/Ang-(1-7)/Mas axis. Naunyn Schmiedebergs Arch Pharmacol 389: 1159-1169, 2016.

10. Tian Y, Li H, Liu P, Xu JM, Irwin MG, Xia Z and Tian G: Captopril pretreatment produces an additive cardioprotection to isoflurane preconditioning in attenuating myocardial ischemia reperfusion injury in rabbits and in humans. Mediators Inflamm 2015: 819232, 2015

11. Lei Y, Xu Q, Zeng B, Zhang W, Zhen Y, Zhai Y, Cheng F, Mei W, Zheng D, Feng J, et al: Angiotensin-(1-7) protects cardiomyocytes against high glucose-induced injuries through inhibiting reactive oxygen species-activated leptin-p38 mitogen-activated protein kinase/extracellular signal-regulated protein kinase $1 / 2$ pathways, but not the leptin-c-Jun N-terminal kinase pathway in vitro. J Diabetes Investig 8: 434-445, 2017.

12. Le Corvoisier P, Adamy C, Sambin L, Crozatier B, Berdeaux A, Michel JB, Hittinger L and Su J: The cardiac renin-angiotensin system is responsible for high-salt diet-induced left ventricular hypertrophy in mice. Eur J Heart Fail 12: 1171-1178, 2010.

13. Idris AH, Becker LB, Ornato JP, Hedges JR, Bircher NG, Chandra NC, Cummins RO, Dick W, Ebmeyer U, Halperin HR, et al: Utstein-style guidelines for uniform reporting of laboratory CPR research. A statement for healthcare professionals from a task force of the American Heart Association, the American College of Emergency Physicians, the American College of Cardiology, the European Resuscitation Council, the Heart and Stroke Foundation of Canada, the Institute of Critical Care Medicine, the Safar Center for Resuscitation Research, and the Society for Academic Emergency Medicine. Writing Group. Circulation 94: 2324-2336, 1996.

14. Pantazopoulos IN, Xanthos TT, Vlachos I, Troupis G, Kotsiomitis E, Johnson E, Papalois A and Skandalakis P: Use of the impedance threshold device improves survival rate and neurological outcome in a swine model of asphyxial cardiac arrest*. Crit Care Med 40: 861-868, 2012.

15. Travers AH, Rea TD, Bobrow BJ, Edelson DP, Berg RA, Sayre MR, Berg MD, Chameides L, O'Connor RE and Swor RA Part 4: CPR overview: 2010 American Heart Association Guidelines for Cardiopulmonary Resuscitation and Emergency Cardiovascular Care. Circulation 122 (18 Suppl 3): S676-S684, 2010.

16. Stadlbauer KH, Rheinberger K, Wenzel V, Raedler C, Krismer AC, Strohmenger HU, Augenstein S, Wagner-Berger HG, Voelckel WG, Lindner KH and Amann A: The effects of nifedipine on ventricular fibrillation mean frequency in a porcine model of prolonged cardiopulmonary resuscitation. Anesth Analg 97: 226-230, 2003.

17. Wang G, Zhang Q, Yuan W, Wu J and Li C: Enalapril protects against myocardial ischemia/reperfusion injury in a swine model of cardiacarrest and resuscitation. Int J Mol Med 38: 1463-1473, 2016.

18. Rezkalla S, Kloner RA, Khatib G and Khatib R: Beneficial effects of captopril in acute coxsakievirus B3 murine myocarditis. Circulation 81: 1039-1046, 1990.

19. Pinsky MR: The right ventricle: Interaction with the pulmonary circulation. Crit Care 20: 266, 2016.
20. Kumamaru KK, George E, Ghosh N, Quesada CG, Wake N, Gerhard-Herman $\mathrm{M}$ and Rybicki FJ: Normal ventricular diameter ratio on $\mathrm{CT}$ provides adequate assessment for critical right ventricular strain among patients with acute pulmonary embolism. Int J Cardiovasc Imaging 32: 1153-1161, 2016.

21. Iwai M and Horiuchi M: Role of the ACE2/angiotensin1-7/Mas axis in the cardiovascular system. Hypertens Res 33: 1108-1109, 2010.

22. Zagorski J, Debelak J, Gellar M, Watts JA and Kline JA: Chemokines accumulate in the lungs of rats with severe pulmonary embolism induced by polystyrene microspheres. J Immunol 171: 5529-5536, 2003.

23. Schultz J, Andersen A, Gade IL, Ringgaard S, Kjaergaard B and Nielsen-Kudsk JE: A porcine in-vivo model of acute pulmonary embolism. Pulm Circ 8: 2045893217738217, 2018.

24. Brain Resuscitation Clinical Trial I Study Group: A randomized clinical study of thiopental loading in comatose survivors of cardiac arrest. N Engl J Med 314: 397-403, 1986.

25. Brain Resuscitation Clinical Trial II Study Group: A randomized clinical study of a calcium-entry blocker(lidoflazine)in the treatment of comatose survivors of cardiac arrest. N Engl J Med 324: I225-1231, 1991

26. Sun XQ, Zhang R, Zhang HD, Yuan P, Wang XJ, Zhao QH, Wang L, Jiang R, Jan Bogaard H and Jing ZC: Reversal of right ventricular remodeling by dichloroacetate is related to inhibition of mitochondria-dependent apoptosis. Hypertens Res 39: 302-311, 2016.

27. Hengartner MO: The biochemistry of apoptosis. Nature 407: 770-776, 2000.

28. Pagliara V, Saide A, Mitidieri E, d'Emmanuele di Villa Bianca R, Sorrentino R, Russo G and Russo A: 5-FU targets rpL3 to induce mitochondrial apoptosis via cystathionine- $\beta$-synthase in coloncancer cells lacking p53. Oncotarget 7: 50333-50348, 2016.

29. Fabris B, Candido R, Bortoletto M, Toffoli B, Bernardi S, Stebel M, Bardelli M, Zentilin L, Giacca M and Carretta R: Stimulation of cardiac apoptosis in ovariectomized hypertensive rats: Potential role of the renin-angiotensin system. J Hypertens 29: 273-281, 2011.

30. Li T, Zhou R, Yao Y, Yang Q, Zhou C, Wu W, Li Q, You Z, Zhao X, Yang L, et al: Angiotensin-converting enzyme inhibitor captopril reverses the adverse cardiovascular effects of polymerized hemoglobin. Antioxid Redox Signal 21: 2095-2108, 2014.

31. Zhao P, Li F, Gao W, Wang J, Fu L, Chen Y and Huang M: Angiotensin 1-7 protects cardiomyocytes from hypoxia/reoxygenation-induced oxidative stress by preventing ROS-associated mitochondrial dysfunction and activating the Akt signaling pathway. Acta Histochem 117: 803-810, 2015.

32. Wan W, Jiang X, Li X, Zhang C and Yi X: Silencing of angiotensin-converting enzyme by RNA interference prevents H9c2 cardiomyocytes from apoptosis induced by anoxia/reoxygenation through regulation of the intracellular renin-angiotensin system. Int J Mol Med 32: 1380-1386, 2013.

33. Qi J, Yu J, Tan Y, Chen R, Xu W, Chen Y, Lu J, Liu Q, Wu J, Gu W and Zhang M: Mechanisms of Chinese Medicine Xinmailong's protection against heart failure in pressure-overloaded mice and cultured cardiomyocytes. Sci Rep 7: 42843, 2017.

34. Li FF, Yuan Y, Liu Y, Wu QQ, Jiao R, Yang Z, Zhou MQ and Tang QZ: Pachymic acid protects H9c2 cardiomyocytes from lipopolysaccharide-induced inflammation and apoptosisby inhibiting the extracellular signal-regulated kinase $1 / 2$ and p38 pathways. Mol Med Rep 12: 2807-2813, 2015

35. Kassiri Z, Zhong J, Guo D, Basu R, Wang X, Liu PP, Scholey JW, Penninger JM and Oudit GY: Loss of angiotensin-converting enzyme 2 accelerates maladaptive left ventricular remodeling in response to myocardial infarction. Circ Heart Fail 2: 446-455, 2009.

This work is licensed under a Creative Commons Attribution-NonCommercial-NoDerivatives 4.0 International (CC BY-NC-ND 4.0) License. 